\title{
Enumeration by Stabilizer Class of Patterns with Local Restrictions
}

\author{
Ifor MorRis and Christopher D. Wensley \\ School of Mathematics \\ University of Wales, Bangor \\ Gwynedd LL57 1UT, U.K. \\ i.morris/c.d.wensley@bangor .ac.uk
}

Submitted: May 24, 1996; Accepted: July 29, 1996.

\begin{abstract}
We consider patterns of colourings of $G$-sets where certain forbidden sub-colourings are excluded. An algorithm is developed for the calculation of inventories of patterns with a prescribed stabilizer class.
\end{abstract}

\section{Introduction}

The theory of pattern enumeration is concerned with a finite group $G$ acting as the group of symmetries of a set $X$, a set of colours $C$, and the action of $G$ on the set $C^{X}$ of colourings of $X$. By a pattern we mean the $G$-orbit of a colouring.

Williamson [6] used a combination of the inclusion - exclusion rule with Redfield/Pólya theory to enumerate patterns of colourings which do not contain colourings of specified subsets of $X$ using chosen subsets of $C$. The aim of this note is to enumerate the subset of these patterns which are stabilized by a chosen conjugacy class of subgroups of $G$.

We use two examples to illustrate the main features of the method. Firstly we discuss a particular example used by Williamson which involves the enumeration of patterns of 8-bead necklaces that do not include particular sub-colourings called a-rooted trees. A second example is taken from the counting of isomers in chemical enumeration. We consider colourings

1991 Mathematics Subject Classification: 05 A15, 05E05.

Keywords: pattern enumeration, inventory, subgroup lattice, isomer. 
of the 8 vertices of a trigonal dodecahedron in which the vertices are of two types, 4-valent and 5-valent, and such that adjacent vertices have different colours. The additional feature here is that excluded colourings may be constructed in different ways from sets of forbidden sub-colourings.

We end by writing down a general algorithm for enumerating inventories of patterns which exclude an arbitrary number of sub-colourings that are specified using an arbitrary partition of the set of colours.

\section{Pattern enumeration}

We first recall some of our notation and results from [4] and [5]. Enumeration by stabilizer class using tables of marks is also discussed in detail in Chapter 3 of [1].

Denote by $\mathcal{L}_{G}$ and $\mathcal{P}_{G}$ the subgroup lattice and the poset of conjugacy classes $[H]$ of subgroups of a group $G$. Let $\mathcal{A}$ be a commutative algebra over the rationals. The incidence algebra $\mathcal{A} I A\left(L_{G}\right)$ has a subalgebra $\mathcal{A} C A\left(L_{G}\right)$ of conjugacy functions satisfying

$$
f(H, K)=f\left(g^{-1} H g, g^{-1} K g\right) \text { for all } H, K \leq G \text { and } g \in G .
$$

There is an algebra homomorphism from $\mathcal{A} C A\left(\mathcal{L}_{G}\right)$ to $\mathcal{A} I A\left(\mathcal{P}_{G}\right)$ which maps $f$ to $\check{f}$ where

$$
\check{f}([H],[K])=\sum_{K^{\prime} \in K} f\left(H, K^{\prime}\right) .
$$

Let $\zeta$ be the zeta function on $\mathcal{L}_{G}$ with inverse the Möbius function $\mu$, and let $\gamma$ be the conjugacy diagonal function defined by $\gamma(K)=|G| /|K||[K]|$. The mark function $\phi \in \mathcal{A} I A\left(\mathcal{P}_{G}\right)$, where $\phi([H],[K])$ is the number of left cosets in $(G / K)$ fixed under left multiplication by $H$, has a factorisation $\phi=\check{\zeta} \gamma$. When a total ordering of the classes $[K]$ has been chosen, compatible with the partial order on $\mathcal{P}_{G}$, the marks form an upper triangular matrix $M(\phi)$, known as the table of marks of $G$, whose inverse is the Burnside matrix $M\left(\phi^{-1}\right)$.

Given a diagonal conjugacy function $\Delta$ on $\mathcal{L}_{G}$ we obtain $\check{\tau}$ from $\alpha$ and $\tau$ where

$$
\alpha=\mu \Delta \zeta, \quad \tau=\gamma^{-1} \alpha \gamma, \quad \check{\tau}=\phi^{-1} \Delta \phi .
$$

The values $\alpha(H, K)$ may be calculated successively, working downwards from $K$ using the intervals $[H, K]$, by the formulae

$$
\alpha(K, K)=\Delta(K), \sum_{H \leq J \leq K} \alpha(H, J)=0 \text { when } H \neq K .
$$

In the case $K=G$,

$$
\check{\tau}([H],[G])=\alpha(H, G) / \gamma(H) .
$$

We now summarise the basic ingredients of generalised Redfield/Pólya enumeration (see, for example, [1], Chapter 2, or [5], Section 5). Let $G$ be a subgroup of the symmetric group $S_{n}$ 
and let $\mathcal{A}=\Lambda_{\ell} \otimes \mathbb{Q}$ where $\Lambda_{\ell}$ is the ring of symmetric polynomials in a set of indeterminates $\Xi_{\ell}=\left\{\xi_{1}, \xi_{2}, \ldots, \xi_{\ell}\right\}$, generated by the power sums $p_{d}=\sum_{i=1}^{\ell} \xi_{i}^{d}$. When we wish to consider two sorts of indeterminates, we write $\Xi_{\ell}=\left\{\eta_{1}, \ldots, \eta_{j}, \theta_{1}, \ldots, \theta_{k}\right\}, j+k=\ell$, and we denote by $q_{d}, r_{d}$ the power sums in $\eta_{i}, \theta_{i}$ respectively, so that $p_{d}=q_{d}+r_{d}$ for all $d \geq 1$.

Let $V$ be a $G$-set and $w: V \rightarrow \mathcal{A}$ a weight function. The weight of a subset $U \subseteq V$ is defined to be $w(U)=\sum_{u \in U} w(u)$. For $g \in G$ and $H \leq G$ we denote the weights of the fixed point subsets $\operatorname{Fix}_{V}(g)$ and $\operatorname{Fix}_{V}(H)$ by $w(V, g)$ and $w(V, H)$ respectively. If $\Omega$ is a transversal for the set of orbits $V / G$ and $w$ is constant on each orbit, the inventory of $V$ is $w(\Omega)$. Each $g \in G$ determines a partition $\pi_{g}=\left(n^{m_{n}} \ldots 2^{m_{2}} 1^{m_{1}}\right)$ of $n$, in which the parts are the lengths of the disjoint cycles, and hence a symmetric function

$$
p_{g}=p_{n}^{m_{n}} \ldots p_{2}^{m_{2}} p_{1}^{m_{1}}=w(V, g) \in \Lambda_{\ell},
$$

the combined weight of all the elements fixed by $g$. The Cauchy-Frobenius Lemma states that

$$
w(\Omega)=\frac{1}{|G|} \sum_{g \in G} p_{g} .
$$

Similarly, each subgroup $H$ of $G$ determines a partition $\pi_{H}=\left(\pi_{1}, \pi_{2}, \ldots, \pi_{h}\right) \vdash n$, in which the parts are the lengths of the orbits, and hence a symmetric function

$$
p_{H}=p_{\pi_{1}} p_{\pi_{2}} \ldots p_{\pi_{h}}=w(V, H) \in \Lambda_{\ell},
$$

the weight of all the elements fixed by $H$.

Defining $\Delta_{p} \in \mathcal{A} C A\left(\mathcal{L}_{G}\right), H \mapsto p_{H}$, we obtain by (1)

$$
\check{\tau}_{p}=\phi^{-1} \Delta_{p} \phi \in \mathcal{A} I A\left(\mathcal{P}_{G}\right) .
$$

(The matrix $M\left(\check{\tau}_{p}\right)$ is the transition matrix between $\beta$-operations and $\lambda$-operations of degree $n$ in a $\beta$-ring (see [5], Corollary 5.2).)

Let $V_{[H]}$ be the subset of $V$ whose elements have stabilizer class $[H]$ and let $\Omega_{[H]}$ be a transversal for $V_{[H]} / G$. The $[H]$-inventories $w\left(\Omega_{[H]}\right)$ of $V$ may be obtained by multiplying the vector whose elements are the values of $\Delta_{p}$ by the Burnside matrix ([1], Section 3.3). Since this vector forms the final column of the matrix $M(\Delta \phi)$, these inventories are the entries $\check{\tau}_{p}([H],[G])$ in the last column of $M\left(\check{\tau}_{p}\right)$.

Now let $X$ be an $n$-element $G$-set, let $C=\left\{c_{1}, c_{2}, \ldots, c_{\ell}\right\}$ be a set of colours and let $w_{C}: C \rightarrow \mathcal{A}, c_{i} \mapsto \xi_{i}$ be a weight function on the colours. The set $V=C^{X}$ of colourings of $X$ has weight function

$$
w: V \rightarrow \mathcal{A}, \quad \chi \mapsto \prod_{x \in X} w_{C}(\chi(x))
$$

Pólya's fundamental theorem states that the inventory of $V$ is the cycle index

$$
\operatorname{Cyc}\left(G ; p_{1}, p_{2}, \ldots, p_{n}\right)=\frac{1}{|G|} \sum_{g \in G} p_{g} \in \mathcal{A} .
$$




\section{Williamson's example}

Given a set $X=\{1,2, \ldots, 8\}$ of 8 beads on a circular necklace, consider the set $V_{0}=C^{X}$ of colourings of the beads using a set of colours $C=A \cup B, A=\left\{a_{1}, a_{2}, \ldots, a_{j}\right\}, B=$ $\left\{b_{1}, b_{2}, \ldots, b_{k}\right\}, A \cap B=\emptyset$. The dihedral group $D_{16}$ acts as the group of symmetries of the uncoloured necklace and has cycle index, expressed as a polynomial in $j=|A|, k=|B|$ :

$$
\operatorname{Cyc}\left(D_{16},\{j, k\}\right)=j^{8}+j^{7} k+4 j^{6} k^{2}+5 j^{5} k^{3}+8 j^{4} k^{4}+5 j^{3} k^{5}+4 j^{2} k^{6}+j k^{7}+k^{8} .
$$

There are 30 patterns when $j=k=1 ; 987$ patterns when $j=2$ and $k=1$; and 7680 patterns when $j=k=2$.

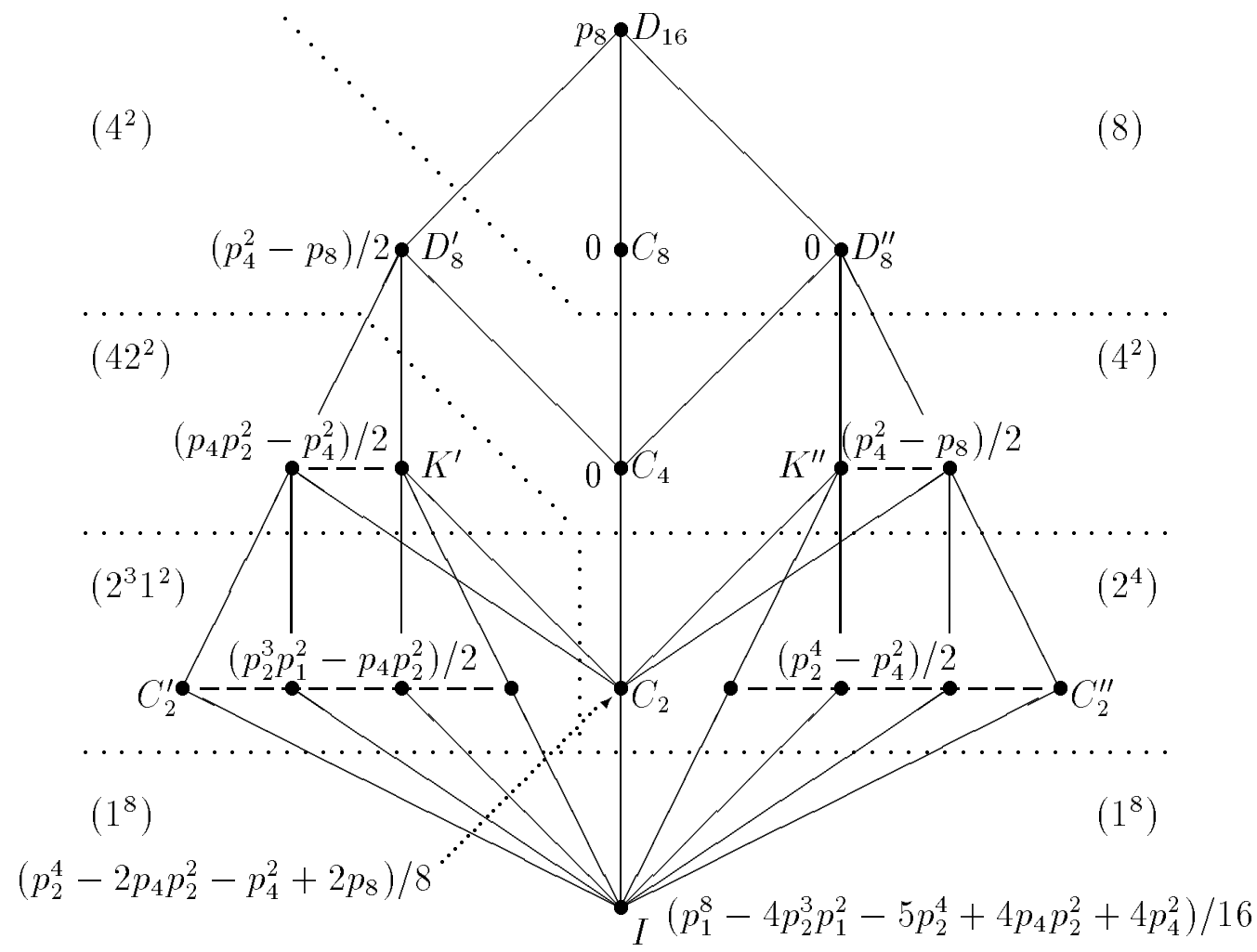

Figure 1: Subgroup Lattice of $D_{16}$ and values $\check{\tau}_{p}\left([H],\left[D_{16}\right]\right)$.

A Hasse diagram for the subgroup lattice of $D_{16}$ is shown in Figure 1, where horizontal dashed lines join the subgroups in a conjugacy class $[H]$. The class $\left[C_{2}^{\prime}\right]$, (resp. $\left[C_{2}^{\prime \prime}\right]$ ) contains those reflections whose axis does (resp. does not) pass through a pair of beads. Dotted lines partition the lattice of $D_{16}$ into subposets in which the subgroups have constant 
orbit type $\pi_{H} \vdash 8$. The poset formed by these regions is isomorphic to the subposet $\left\{\pi_{H} \mid H \leq\right.$ $\left.D_{16}\right\}$ of the poset of all partitions of 8 . Values $\check{\tau}_{p}\left([H],\left[D_{16}\right]\right)$ are shown for each conjugacy class $[H]$. Note that $\check{\tau}_{p}\left([H],\left[D_{16}\right)=0\right.$ when the subgroups in $[H]$ are not maximal in the subposet of type $\pi_{H}$.

Summing over all subgroup classes, we obtain the cycle index:

$$
\sum_{H \leq D_{16}} \check{\tau}_{p}\left([H],\left[D_{16}\right]\right)=\operatorname{Cyc}\left(D_{16} ; p_{1}, \ldots, p_{8}\right)=\frac{1}{16}\left(p_{1}^{8}+4 p_{2}^{3} p_{1}^{2}+5 p_{2}^{4}+2 p_{4}^{2}+4 p_{8}\right) .
$$

When $\Delta$ is an arbitrary diagonal function, constant on these subposets, the non-zero values of $\check{\tau}\left([H],\left[D_{16}\right]\right)$ are given in Table 1 .

\begin{tabular}{|c|l|}
\hline$H$ & $\check{\tau}\left([H],\left[D_{16}\right]\right)$ \\
\hline$D_{16}$ & $\Delta\left(D_{16}\right)$ \\
$D_{8}^{\prime}$ & $\left(\Delta\left(D_{8}^{\prime}\right)-\Delta\left(D_{16}\right)\right) / 2$ \\
$K^{\prime \prime}$ & $\left(\Delta\left(K^{\prime \prime}\right)-\Delta\left(D_{16}\right)\right) / 2$ \\
$K^{\prime}$ & $\left(\Delta\left(K^{\prime}\right)-\Delta\left(D_{8}^{\prime}\right)\right) / 2$ \\
$C_{2}^{\prime \prime}$ & $\left(\Delta\left(C_{2}^{\prime \prime}\right)-\Delta\left(K^{\prime \prime}\right)\right) / 2$ \\
$C_{2}^{\prime}$ & $\left(\Delta\left(C_{2}^{\prime}\right)-\Delta\left(K^{\prime}\right)\right) / 2$ \\
$C_{2}$ & $\left(\Delta\left(C_{2}\right)-2 \Delta\left(K^{\prime}\right)-2 \Delta\left(K^{\prime \prime}\right)+\Delta\left(D_{8}^{\prime}\right)+2 \Delta\left(D_{16}\right)\right) / 8$ \\
$I$ & $\left(\Delta(I)-\Delta\left(C_{2}\right)-4 \Delta\left(C_{2}^{\prime}\right)-4 \Delta\left(C_{2}^{\prime \prime}\right)+4 \Delta\left(K^{\prime}\right)+4 \Delta\left(K^{\prime \prime}\right)\right) / 16$ \\
\hline
\end{tabular}

Table 1: Values of $\check{\tau}$ for arbitrary $\Delta$.

Williamson's problem was to determine the number of patterns which do not contain an $a$-rooted tree, namely a coloured subpattern of the form marked in Figure 2.

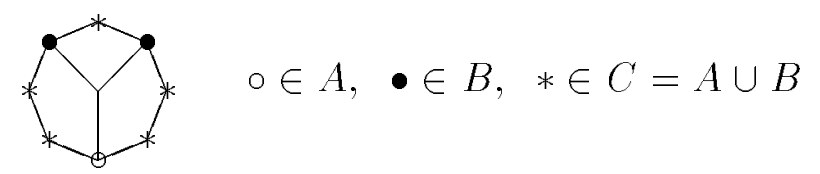

Figure 2: A necklace containing an $a$-rooted tree.

In the case $\ell=2, \Xi=\{\eta, \theta\}$, rewriting the $\check{\tau}_{p}\left([H],\left[D_{16}\right]\right)$ we obtain symmetric functions in $\eta$ and $\theta$ as listed in Table 2. The 30 monomials correspond to the 30 colourings of the necklace with the set $\{a, b\}$. Just 13 of these have no $a$-rooted tree ([6], Figure 4).

Let $P=\left\{P_{1}, P_{2}, \ldots, P_{8}\right\}$ be the set of properties such that $\chi \in V_{0}$ satisfies $P_{i}$ if $\chi$ contains an $a$-rooted tree with bead $i$ as root. For $Q \subseteq P$ let

$$
V_{Q}=\left\{\chi \in V_{0} \mid \chi \text { satisfies } P_{i} \text { for all } P_{i} \in Q\right\}
$$




\begin{tabular}{|c|c|l|}
\hline$(8)$ & $D_{16}$ & $\eta^{8}+\theta^{8}$ \\
$\left(4^{2}\right)$ & $D_{8}^{\prime}$ & $\eta^{4} \theta^{4}$ \\
$\left(4^{2}\right)$ & $K^{\prime \prime}$ & $\eta^{4} \theta^{4}$ \\
$\left(42^{2}\right)$ & $K^{\prime}$ & $\eta^{6} \theta^{2}+\eta^{2} \theta^{6}$ \\
$\left(2^{4}\right)$ & $C_{2}^{\prime \prime}$ & $2\left(\eta^{6} \theta^{2}+\eta^{2} \theta^{6}\right)+2 \eta^{4} \theta^{4}$ \\
$\left(2^{3} 1^{2}\right)$ & $C_{2}^{\prime}$ & $\left(\eta^{7} \theta+\eta \theta^{7}\right)+\left(\eta^{6} \theta^{2}+\eta^{2} \theta^{6}\right)+3\left(\eta^{5} \theta^{3}+\eta^{3} \theta^{5}\right)+2 \eta^{4} \theta^{4}$ \\
$\left(1^{8}\right)$ & $I$ & $2\left(\eta^{5} \theta^{3}+\eta^{3} \theta^{5}\right)+2 \eta^{4} \theta^{4}$ \\
\hline
\end{tabular}

Table 2: Non-zero values of $\check{\tau}_{p}\left([H],\left[D_{16}\right]\right)$ when $\ell=2$ (30 terms).

We represent the elements of $V_{Q}$ by colourings having a marked $a$-rooted tree at each bead $i$ for which $P_{i} \in Q$. If $V_{f}$ is the set of colourings with no a-rooted tree then, using the principle of inclusion-exclusion, we define $\Delta_{f} \in \mathcal{A} C A\left(\mathcal{L}_{G}\right)$ by

$$
\Delta_{f}(H)=w\left(V_{f}, H\right)=\sum_{Q \subseteq P}(-1)^{|Q|} w\left(V_{Q}, H\right) .
$$

If a colouring $\chi$ contains $e$ a-rooted trees, then $\chi$ belongs to $2^{e}$ of the subsets $V_{Q}$ and, if fixed by $H$, contributes $(1-1)^{e}=0$ to the sum (4). Only 47 of the 256 subsets $V_{Q}$ are non-empty. For example, $V_{\left\{P_{1}, P_{4}\right\}}=\emptyset$ since properties $P_{1}$ and $P_{4}$ require beads 1 and 4 to be coloured with both $A$ and $B$.

The first stage of the solution is to produce a complete set of marked template patterns $T_{t}$ containing all possible arrangements of one or more $a$-rooted trees, modulo the group action. Such templates may be considered as patterns using $\{a, b, c\}$ as the set of colours: beads in the marked trees are coloured $a$ (for the root) or $b$, the remaining beads are coloured $c$. In simple cases the templates can be found by inspection. In general, Williamson's multilinear techniques ([6], Section 2) may be used to construct them.
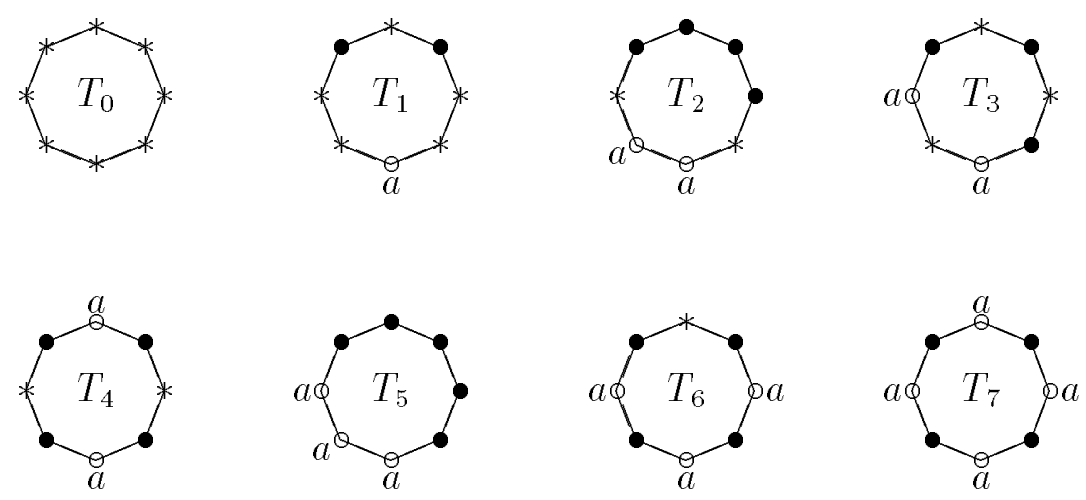

Figure 3: The 8 template patterns $T_{t}$.

The 8 templates for our example are shown in Figure 3, where $\circ, \bullet, *$ denote vertices 
coloured $a, b, c$ respectively. Since an $a$-rooted tree is uniquely determined by the location of its root, we mark each tree by placing an $a$ next to the root bead. Denoting by $\nu_{t}$ the number of $a$-rooted trees in $T_{t}$, we have $\nu_{0}=0, \nu_{1}=1, \nu_{2}=\nu_{3}=\nu_{4}=2, \nu_{5}=\nu_{6}=3$ and $\nu_{7}=4$.

The stabilizer classes for these templates are given in Table 3.

\begin{tabular}{|r||rrrrrrrr|}
\hline Template & $T_{0}$ & $T_{1}$ & $T_{2}$ & $T_{3}$ & $T_{4}$ & $T_{5}$ & $T_{6}$ & $T_{7}$ \\
\hline Size of orbit & 1 & 8 & 8 & 8 & 4 & 8 & 8 & 2 \\
\hline Stabilizer class & $D_{16}$ & $C_{2}^{\prime}$ & $C_{2}^{\prime \prime}$ & $C_{2}^{\prime}$ & $K^{\prime}$ & $C_{2}^{\prime}$ & $C_{2}^{\prime}$ & $D_{8}^{\prime}$ \\
\hline
\end{tabular}

Table 3: Stabilizers of the templates.

For $\psi$ a template colouring in $T_{t}$, partition $X$ into subsets $X_{\psi a}, X_{\psi b}, X_{\psi c}$ of beads coloured $a, b, c$ respectively. A marked colouring of type $T_{t}$ is obtained by recolouring the beads in $X_{\psi a}, X_{\psi b}, X_{\psi c}$ with colours from $A, B, C$ respectively. If $V_{t}$ denotes the set of marked colourings of type $T_{t}$ then

$$
\left|V_{t}\right|=\left|T_{t}\right| j^{\left|X_{\psi a}\right|} k^{\left|X_{\psi b}\right|} \ell^{\left|X_{\psi c}\right|}
$$

Since these template colourings include exactly the 47 non-empty $V_{Q}$, equation (4) may be rewritten as

$$
\Delta_{f}(H)=\sum_{t=0}^{7}(-1)^{\nu_{t}} w\left(V_{t}, H\right) .
$$

In our example $\left|V_{1}\right|=8 j k^{2} \ell^{5}$ so when $j=k=1$ there are 256 colourings with a single marked $a$-rooted tree. These colourings form 30 marked patterns and in Figure 4 we give a transversal for $V_{1} / D_{16}$, where $\sim$ indicates different markings of the same colouring. (It is a coincidence that $V_{0} / D_{16}$ contains 30 patterns and $V_{1} / D_{16}$ contains 30 marked patterns.)

Redefine the weight of each colour by

$$
w_{C}: C \rightarrow \mathcal{A}, a_{i} \mapsto \eta_{i}(1 \leq i \leq j), \quad b_{i} \mapsto \theta_{i}(1 \leq i \leq k)
$$

Let $\Omega_{t}$ be a transversal for the patterns $V_{t} / D_{16}$. For each class $[H]$ we require the $[H]$ inventory $w\left(\Omega_{t}\right)$ as a polynomial in the power sums $p_{d}, q_{d}$ and $r_{d}$. Let $X / H=\left\{O_{1}, \ldots, O_{h}\right\}$ and let the orbit $O_{i}$ contain $d_{i}$ beads. Let $V_{\psi}$ be the set of marked colourings of type $T_{t}$ obtained from $\psi \in T_{t}$. Just as $p_{H}$ is a product of power sums, one for each orbit, so the total weight $w\left(V_{\psi}, H\right)$ of colourings fixed by $H$ is a product of $h$ factors. If $O_{i}$ contains a bead coloured $a$ and a bead coloured $b$ then $\operatorname{Fix}_{V_{\psi}}(H)$ is empty and $w\left(V_{\psi}, H\right)=0$. If $O_{i}$ contains only vertices in $X_{\psi_{c}}$ then $O_{i}$ contributes a factor $p_{d_{i}}=\left(q_{d_{i}}+r_{d_{i}}\right)$. If $O_{i}$ only contains vertices from $X_{\psi a} \cup X_{\psi c}$ (resp. $X_{\psi b} \cup X_{\psi c}$ ) then $O_{i}$ contributes $q_{d_{i}}$ (resp. $r_{d_{i}}$ ). We thus obtain $\Delta_{t} \in \mathcal{A} I A\left(\mathcal{P}_{G}\right)$ where

$$
\Delta_{t}([H])=w\left(V_{t}, H\right)=\sum_{\psi \in T_{t}} w\left(V_{\psi}, H\right) \in \mathcal{A} .
$$



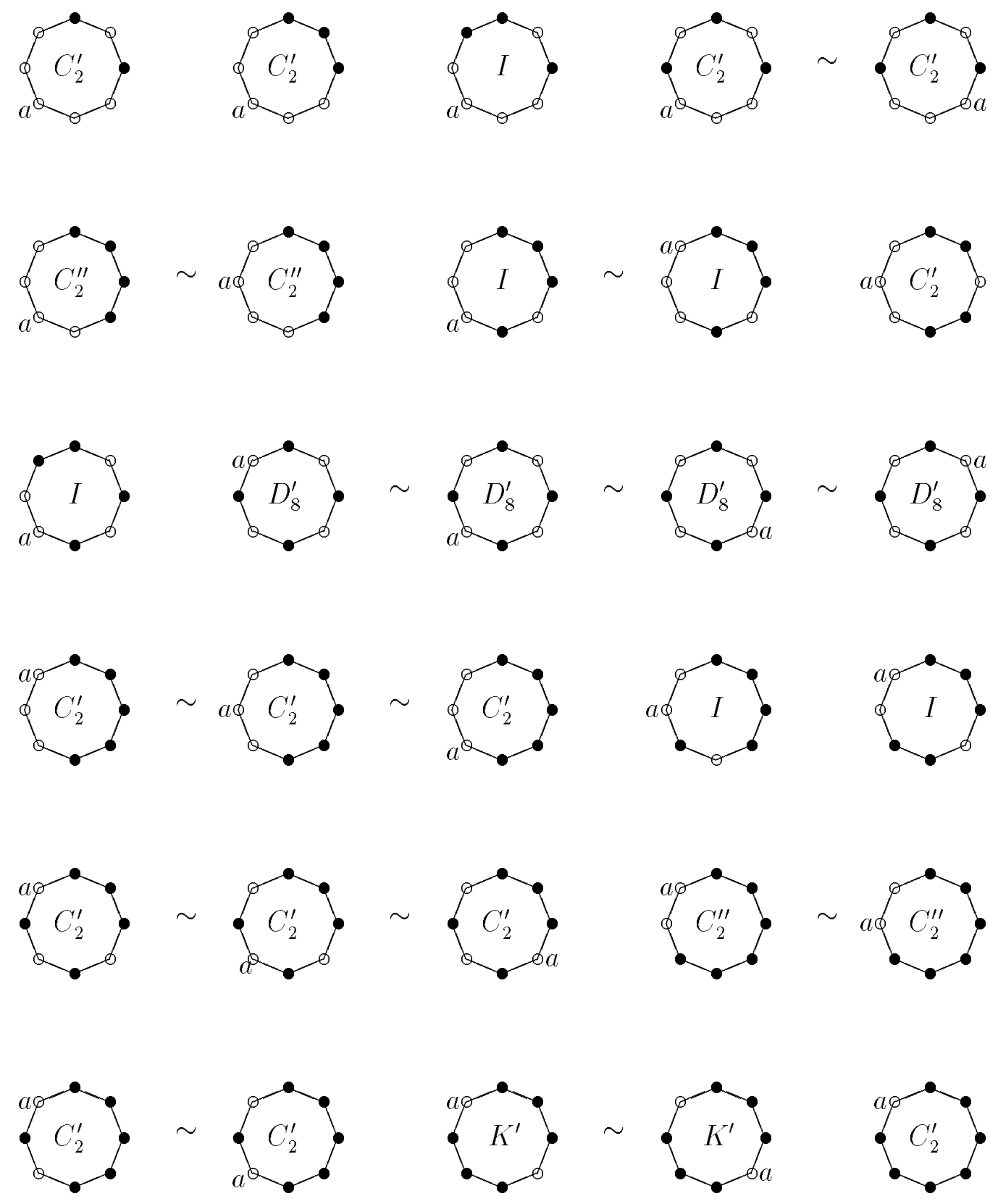

Figure 4: The 30 marked patterns of type $T_{1}$ and their stabilizer classes. 
Reversing the order of summation in $\sum_{H^{\prime} \in[H]} \sum_{\psi \in T_{t}} w\left(V_{\psi}, H^{\prime}\right)$, we obtain an alternative formula

$$
\Delta_{t}([H])=\frac{\left|T_{t}\right|}{|[H]|} \sum_{H^{\prime} \in[H]} w\left(V_{\psi}, H^{\prime}\right)
$$

which is more convenient when the size of $[H]$ is small compared with that of $T_{t}$. Since $\Delta_{t}$ is a conjugacy function we may define $\check{\tau}_{t}=\phi^{-1} \Delta_{t} \phi$ and so $\check{\tau}_{t}\left([H],\left[D_{16}\right]\right)$ is the inventory $w\left(\Omega_{t}\right)$.

Equations (4) and (5) define a conjugacy function $\Delta_{f}$ where $\Delta_{f}(H)$ is the total weight of the colourings which are free of $a$-rooted trees. The corresponding $\check{\tau}_{f}$ is given by a similar inclusion-exclusion formula:

$$
\check{\tau}_{f}=\phi^{-1} \Delta_{f} \phi=\phi^{-1} \sum_{t=0}^{7}(-1)^{\nu_{t}} \Delta_{t} \phi=\sum_{t=0}^{7}(-1)^{\nu_{t}} \phi^{-1} \Delta_{t} \phi=\sum_{t=0}^{7}(-1)^{\nu_{t}} \check{\tau}_{t} .
$$

and $w\left(\Omega_{f,[H]}\right)=\check{\tau}_{f}\left([H],\left[D_{16}\right]\right)$ where $\Omega_{f}$ is a transversal for patterns with no $a$-rooted tree.

\begin{tabular}{|c|l|}
\hline$H$ & $\Delta_{f}(H)$ \\
\hline$D_{16}, D_{8}^{\prime \prime}, C_{8}$ & $p_{8}$ \\
$D_{8}^{\prime}, C_{4}$ & $p_{4}^{2}-2 q_{4} r_{4}$ \\
$K^{\prime \prime}$ & $p_{4}^{2}$ \\
$K^{\prime}$ & $p_{4} p_{2}^{2}-2 p_{2} q_{2} r_{4}+q_{2}^{2} r_{4}-q_{4} r_{2}^{2}$ \\
$C_{2}^{\prime \prime}$ & $p_{2}^{4}-2 p_{2} q_{2} r_{2}^{2}$ \\
$C_{2}^{\prime}$ & $p_{2}^{3} p_{1}^{2}-2 p_{2}^{2} p_{1} q_{1} r_{2}-2 p_{2} p_{1} q_{2} r_{2} r_{1}+p_{2} q_{1}^{2} r_{2}^{2}-p_{1}^{2} q_{2} r_{2}^{2}+2 p_{1} q_{2} q_{1} r_{2}^{2}$ \\
& $+2 q_{2} r_{2}^{2} r_{1}+q_{2}^{2} r_{2} r_{1}^{2}-q_{2} q_{1}^{2} r_{2}^{2}$ \\
$C_{2}$ & $p_{2}^{4}-4 p_{2} q_{2} r_{2}^{2}+2 q_{2}^{2} r_{2}^{2}$ \\
$I$ & $p_{1}^{8}-8 p_{1}^{5} q_{1} r_{1}^{2}+12 p_{1}^{2} q_{1}^{2} r_{1}^{4}+8 p_{1}^{3} q_{1}^{2} r_{1}^{3}-8 p_{1} q_{1}^{3} r_{1}^{4}-8 q_{1}^{3} r_{1}^{5}+2 q_{1}^{4} r_{1}^{4}$ \\
\hline
\end{tabular}

Table 4: Weights of colourings with no $a$-rooted tree.

The values of $\Delta_{f}$ are given in Table 4 and may be substituted in Table 1 to give the $[H]$-inventories for $\Omega_{f}$. The first four of these contain relatively few terms:

$$
\begin{aligned}
\check{\tau}_{f}\left(\left[D_{16}\right],\left[D_{16}\right]\right) & =p_{8}=\Sigma \eta_{1}^{8}+\Sigma \theta_{1}^{8} \\
\check{\tau}_{f}\left(\left[D_{8}^{\prime}\right],\left[D_{16}\right]\right) & =\left(p_{4}^{2}-p_{8}\right) / 2-q_{4} r_{4}=\Sigma \eta_{1}^{4} \eta_{2}^{4}+\Sigma \theta_{1}^{4} \theta_{2}^{4} \\
\check{\tau}_{f}\left(\left[K^{\prime \prime}\right],\left[D_{16}\right]\right) & =\left(p_{4}^{2}-p_{8}\right) / 2=\Sigma \eta_{1}^{4} \eta_{2}^{4}+\Sigma \eta_{1}^{4} \theta_{1}^{4}+\Sigma \theta_{1}^{4} \theta_{2}^{4} \\
\check{\tau}_{f}\left(\left[K^{\prime}\right],\left[D_{16}\right]\right) & =q_{4}\left(q_{2}^{2}-q_{4}\right) / 2+q_{4} q_{2} r_{2}+r_{4}\left(r_{2}^{2}-r_{4}\right) / 2 \\
& =\Sigma \eta_{1}^{6} \eta_{2}^{2}+\Sigma \eta_{1}^{4} \eta_{2}^{2} \eta_{3}^{2}+\Sigma \eta_{1}^{6} \theta_{1}^{2}+\Sigma \eta_{1}^{4} \eta_{2}^{2} \theta_{1}^{2}+\Sigma \theta_{1}^{6} \theta_{2}^{2}+\Sigma \theta_{1}^{4} \theta_{2}^{2} \theta_{3}^{2}
\end{aligned}
$$

\section{The trigonal dodecahedron}

In [2] and [3] Lloyd has applied Redfield/Pólya methods to the enumeration of chemical isomers. In this situation the $G$-set $X=\{1, \ldots, 8\}$ is a molecule whose elements are termed sites, and these sites are 'coloured' with ligands which are groupings of one or more atoms. 


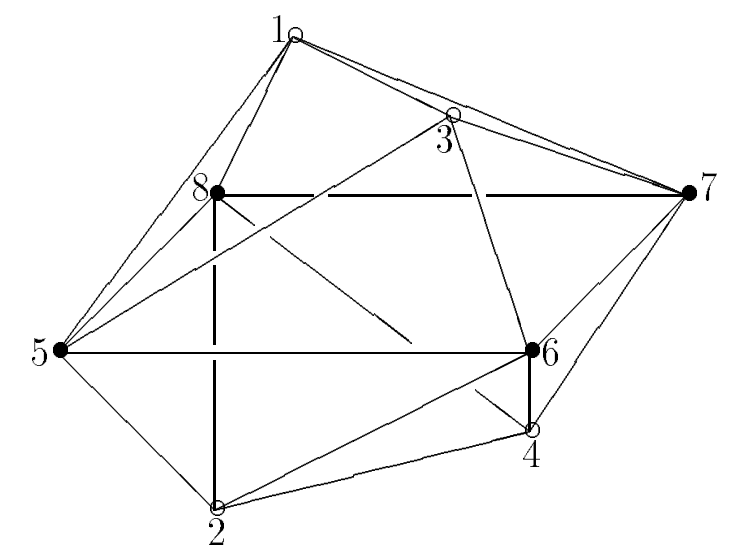

Figure 5: The trigonal dodecahedron.

There are chemical compounds which have eight ligands situated at the vertices of a trigonal dodecahedron (see Figure 5). The rotation group of the polyhedron contains three half-turns about mutually perpendicular axes, and the full rotation group is isomorphic to the dihedral group $D_{8}$ with elements

$$
\begin{array}{cccc}
(), & (13)(24)(57)(68), & (12)(34)(58)(67), & (14)(23)(56)(78), \\
(13)(68), & (24)(57), & (1432)(5876), & (1234)(5678) .
\end{array}
$$

Since four of the sites are 4 -valent and the remainder 5 -valent, we colour $\{1,2,3,4\}$ with a set of ligands $A$ and $\{5,6,7,8\}$ with a second set $B$. The appropriate cycle index is therefore

$$
\operatorname{Cyc}\left(D_{8},\left\{q_{i}, r_{i} \mid 1 \leq i \leq 4\right\}\right)=\frac{1}{8}\left(q_{1}^{4} r_{1}^{4}+2 q_{2} q_{1}^{2} r_{2} r_{1}^{2}+3 q_{2}^{2} r_{2}^{2}+2 q_{4} r_{4}\right)
$$

We suppose, as before, that $|A|=j,|B|=k$, and consider the enumeration of nonadjacent colourings $\chi$ : colourings such that no two adjacent sites contain the same ligand. In this example the set of properties is $P=\left\{P_{13}, P_{24}, P_{56}, P_{67}, P_{78}, P_{58}\right\}$ where $\chi$ satisfies $P_{i h}$ if sites $i$ and $h$ contain the same ligand. First we restrict to the subset $P_{A}=\left\{P_{13}, P_{24}\right\}$, and call $\chi$ A-adjacent if $\chi(1)=\chi(3)$ or $\chi(2)=\chi(4)$. Then we apply the restriction to the square $\{5,6,7,8\}$, using $P_{B}=\left\{P_{56}, P_{67}, P_{78}, P_{58}\right\}$.

The only adjacent pairs of sites coloured with $A$ are $\{1,3\}$ and $\{2,4\}$. Fix $a_{i} \in A$ and partition $A$ as $\left\{a_{i}\right\} \cup \bar{A}_{i}$. There are only four types of marked template pattern, colouring $X$ with $\left\{a_{i}, a_{h}, a, b\right\}$, as shown in Figure 6 .

The inclusion-exclusion formula for the weight of the set $A_{f}$ of colourings which are not $A$-adjacent is

$$
\Delta_{A_{f}}=\Delta_{0}-\sum_{i=1}^{j} \Delta_{1 i}+\sum_{i=1}^{j} \Delta_{2 i}+\sum_{i \neq h} \Delta_{2 i h} .
$$



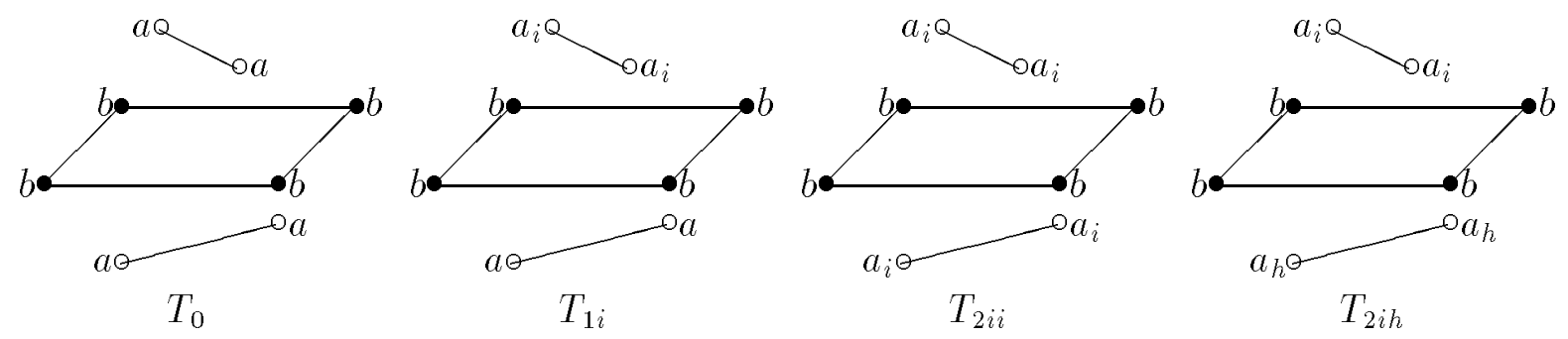

Figure 6: $A$-adjacent templates.

The individual $w\left(V_{t}, H\right)$ need not be symmetric functions, but become so under summation, as in

$$
\sum_{i=1}^{j} w\left(V_{2 i h}, K\right)=\sum_{i \neq h} \eta_{i}^{2} \eta_{h}^{2} r_{2}^{2}=\frac{1}{2}\left(q_{2}^{2}-q_{4}\right) r_{2}^{2} .
$$

The only non-zero weights and $[H]$-enumerators are shown in Table 5.

\begin{tabular}{|c|c|c|}
\hline$[H]$ & $\Delta_{A_{f}}(H)$ & $\check{\tau}_{A_{f}}\left([H],\left[D_{16}\right]\right)$ \\
\hline$C_{2}^{\prime \prime}$ & $\left(q_{2}^{2}-q_{4}\right) r_{2}^{2}$ & $\left(q_{2}^{2}-q_{4}\right) r_{2}^{2} / 2$ \\
$I$ & $\left(q_{1}^{2}-q_{2}\right)^{2} r_{1}^{4}$ & $\left(\left(q_{1}^{2}-q_{2}\right)^{2} r_{1}^{4}-2\left(q_{2}^{2}-q_{4}\right) r_{2}^{2}\right) / 8$ \\
\hline
\end{tabular}

Table 5: Enumerators of non- $A$-adjacent patterns.

Now consider colourings in which adjacent sites among $\{5,6,7,8\}$ contain different ligands but with no restriction on $\{1,2,3,4\}$. The marked templates are shown in Figure 7.
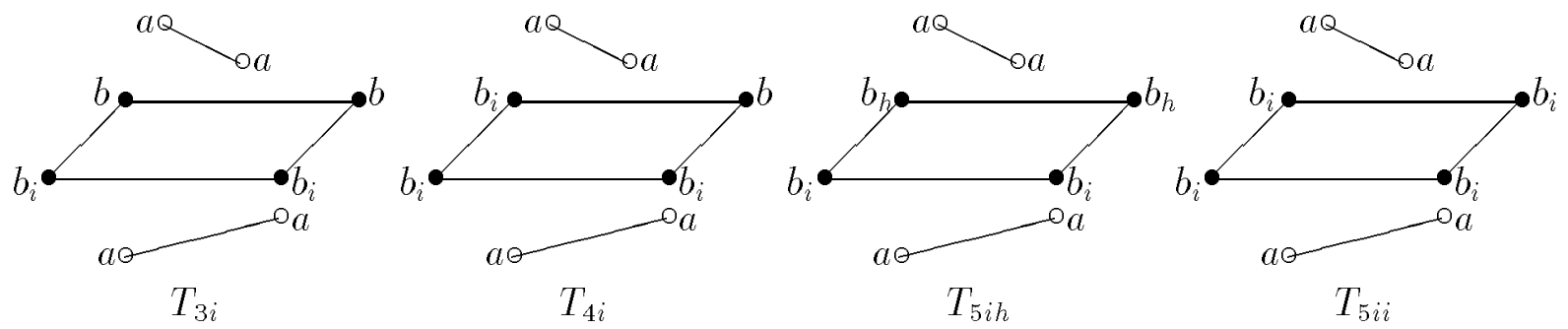

Figure 7: B-adjacent templates.

Because each site belongs to two adjacent pairs it is not the case, as in Williamson's example, that distinct $Q, Q^{\prime} \subseteq P$ always give rise to different marked templates. In fact subsets 


$$
\begin{aligned}
& \left\{P_{56}, P_{78}\right\},\left\{P_{67}, P_{58}\right\}, \\
& \left\{P_{56}, P_{67}, P_{78}\right\},\left\{P_{56}, P_{67}, P_{58}\right\},\left\{P_{56}, P_{78}, P_{58}\right\},\left\{P_{67}, P_{78}, P_{58}\right\}, \\
& \left\{P_{56}, P_{67}, P_{78}, P_{58}\right\}
\end{aligned}
$$

all give rise to marked templates $T_{5 i i}$. Thus, when converting the general inclusion-exclusion formula (4) to a formula involving the $\Delta_{t}$, the coefficient of $\Delta_{5 i i}$ is $2-4+1=-1$. The full formula is:

$$
\Delta_{B_{f}}=\Delta_{0}-\sum_{i=1}^{k} \Delta_{3 i}+\sum_{i=1}^{k} \Delta_{4 i}+\sum_{i \neq h} \Delta_{5 i h}-\sum_{i=1}^{k} \Delta_{5 i i} .
$$

The only non-zero weights are

$$
\begin{aligned}
\Delta_{B_{f}}(K)=\Delta_{B_{f}}\left(C_{2}\right) & =q_{2}^{2}\left(r_{2}^{2}-r_{4}\right) \\
\Delta_{B_{f}}\left(C_{2}^{\prime \prime \prime}\right) & =q_{2} q_{1}^{2}\left(r_{2} r_{1}^{2}-2 r_{3} r_{1}+r_{4}\right), \\
\Delta_{B_{f}}(I) & =q_{1}^{4}\left(r_{1}^{4}-4 r_{2} r_{1}^{2}+4 r_{3} r_{1}+2 r_{2}^{2}-3 r_{4}\right),
\end{aligned}
$$

where the subgroups in class $\left[C_{2}^{\prime \prime \prime}\right]$ are generated by the reflections in the planes $1,3,6,8$ and $2,4,5,7$ and $K$ is their join. The non-zero $[H]$-enumerators for $B$-adjacent free patterns are

$$
\begin{aligned}
\check{\tau}_{B_{f}}\left([K],\left[D_{8}\right]\right) & =\Delta_{B_{f}}(K) / 2 \\
\check{\tau}_{B_{f}}\left(\left[C_{2}^{\prime \prime \prime}\right],\left[D_{8}\right]\right) & =\left(\Delta_{B_{f}}\left(C_{2}^{\prime \prime \prime}\right)-\Delta_{B_{f}}(K)\right) / 2 \\
\check{\tau}_{B_{f}}\left([I],\left[D_{8}\right]\right) & =\left(\Delta_{B_{f}}(I)-2 \Delta_{B_{f}}\left(C_{2}^{\prime \prime \prime}\right)+\Delta_{B_{f}}(K)\right) / 8
\end{aligned}
$$

In order to deal with non-adjacent colourings, we should need to consider all possible adjacencies, leading to a total of 20 marked templates. We omit the details.

\section{The general algorithm}

The following algorithm applies to an enumeration problem interpreted as the counting of patterns of valid colourings having a chosen stabilizer class $[H]$ in a group $G$. Colourings are invalid if they satisfy one or more of a set $P=\left\{P_{1}, P_{2}, \ldots, P_{\lambda}\right\}$ of forbidden properties. These properties may be expressed in terms of a partition $/ A_{1} / A_{2} / \ldots / A_{\kappa} /$ of a set of colours $C$. If $A_{i}=\left\{a_{i 1}, a_{i 2}, \ldots, a_{i j_{i}}\right\}$ and $\sum_{i=1}^{\kappa} j_{i}=\ell$, take $\Xi$ to be a set of $\ell$ indeterminates $\left\{\eta_{i h} \mid 1 \leq i \leq \kappa, 1 \leq h \leq j_{i}\right\}$ and let $w_{c}: C \rightarrow \mathcal{A}, a_{i h} \mapsto \eta_{i h}$, be the corresponding weight function. Denote by $q_{i d}$ the power sum $\sum_{h=1}^{j_{i}} \eta_{i h}^{d}$ and by $V_{f}$ the set of valid colourings.

\section{Algorithm 5.1}

1. Use Williamson's multilinear techniques to obtain a complete set of marked template patterns $T_{t}$ coloured with a set of colours $\left\{c_{1}, c_{2}, \ldots, c_{\kappa}\right\}$.

2. For each template $T_{t}$ and for each $[H] \leq[K] \leq[G]$ determine, using formulae $(6)$ and (7), the weights $\Delta_{t}([K])=w\left(V_{t}, K\right)$ as polynomials in the $q_{i d}$. 
3. Determine the total weight $\Delta_{f}([K])$ of colourings in $V_{f}$ fixed by $K$ by rewriting the inclusion-exclusion formula (4) for the $\lambda$ properties as a signed sum of the $\Delta_{t}([K])$.

4. Calculate values of $\alpha_{f}=\mu \Delta_{f} \zeta$ and $\check{\tau}_{f}=\phi^{-1} \Delta_{f} \phi$ using equations (2) and (3), working down from $[G]$ over the interval $[[H],[G]]$ of $\mathcal{P}_{G}$.

Theorem 5.2 The polynomial $\check{\tau}_{f}([H],[G])$ is the $[H]$-inventory for patterns of valid colourings. When this polynomial is expressed as a polynomial in the indeterminates $\eta_{i h}$, the coefficient of $\prod \eta_{i h}^{d_{i h}}$ is the number of patterns in which colour $a_{i h}$ is used $d_{i h}$ times.

\section{References}

[1] Kerber, A., Algebraic combinatorics via finite group actions, (Bibliographisches Institut Wissenschaftsverlag, Mannheim, 1991).

[2] Lloyd, E.K., Redfield's papers and their relevance to counting isomers and isomerizations, Discrete Applied Math. 19 (1988) 289-304.

[3] Lloyd, E.K., Marks of permutation groups and isomer enumeration, J. Math. Chemistry 11 (1992) 207-222.

[4] Morris, I. And Wensley, C.D. Adams operations and $\lambda$-operations in $\beta$-rings, Discrete Math. 50 (1984) 253-270.

[5] Morris, I. And Wensley, C.D., Cycle indices and subgroup lattices, Discrete Math. 118 (1993) 173-195.

[6] Williamson, S.G., The combinatorial analysis of patterns and the principle of inclusion-exclusion, Discrete Math. 1 (1972) 357-388. 